\title{
X-BAND DIPOLE MODE DEFLECTING CAVITY FOR THE UCLA NEPTUNE BEAMLINE*
}

\author{
R. J. England, B. O’Shea, J. B. Rosenzweig, G. Travish, UCLA, Los Angeles, CA 90095, USA \\ D. Alesini, INFN/LNF, Frascati, Italy
}

\begin{abstract}
We report progress on the design and construction of a nine-cell cavity operating in a $T M_{110}$-like dipole mode for use as a temporal diagnostic of the $14 \mathrm{MeV}, 300 \mathrm{pC}$ electron bunches generated at the UCLA Neptune Laboratory linear accelerator, with an anticipated temporal resolution of $50 \mathrm{fs}$ at a peak input power of $50 \mathrm{~kW}$. The cavity is a center-fed standing-wave pi-mode structure, operating at 9.6 GHz, and incorporating a knife-edge and gasket assembly which minimizes the need for brazing or welding. Results of initial RF testing of the prototype cavity are discussed and compared with simulation results obtained using the commercial code HFSS.
\end{abstract}

\section{INTRODUCTION}

In a traditional linear RF-based accelerator, the electromagnetic mode utilized to accelerate the beam is designed to give rise to a longitudinal electric field with zero transverse deflection for a beam traveling on the axis. Although some transverse fields may be present on-axis near the coupling irises connecting the cells, in the case of a multicell structure or disk-loaded waveguide, the fields generated in the central regions of the cells typically resemble the $T M_{0 n 0}$ modes of a pillbox structure. Modes which resemble the $T M_{1 n 0}$ dipole modes are avoided for purposes of longitudinal acceleration since they produce a strong transverse deflection of the beam. Deflecting mode structures have long been used, however, for particle separation in beams of more than one species $[1,2]$ and as a longitudinal bunch length diagnostic [3]. Some recent efforts have focused on longitudinal phase space reconstruction $[4,5]$ and creation of temporal correlations for X-ray compression [6].

Recent bunch-shaping experiments at the UCLA Neptune Laboratory [7] require the implementation of a beam diagnostic capable of resolving the temporal structure of the sub-picosecond to several picosecond duration electron bunches generated by the Neptune linear accelerator beamline and dogleg compressor with resolutions at the $50 \mathrm{fs}$ level. For this purpose, we have designed a 9-cell standingwave deflecting mode cavity operating at an X-Band frequency of $9.59616 \mathrm{GHz}$. A diagram of the proposed diagnostics section with the deflecting cavity in place is shown in Fig. 1.

${ }^{*}$ Work supported by U.S. Department of Energy under Grant No. DEFG03-92ER40693

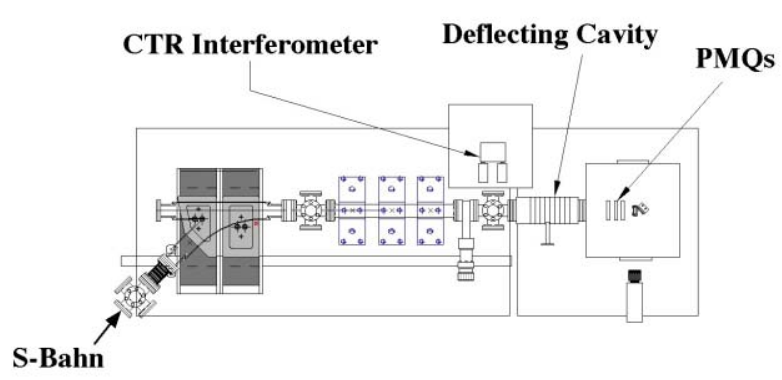

Figure 1: Layout of proposed diagnostics section with table of experimental parameter goals.

\section{CAVITY DESIGN}

The proposed diagnostic for measuring the longitudinal current profile of the compressed bunches produced by the Neptune linear accelerator and dogleg compressor is a linearly polarized $T M_{110}$ dipole mode cavity, being developed in collaboration with the INFN Laboratori Nazionali di Frascati. By correctly designing the input power coupler, the polarity of the transverse magnetic field in this mode may be selected to correspond with either the $x$ or $y$ axis of the beamline. When a beam is injected into such a cavity at the zero-crossing of the RF, it experiences a transverse momentum kick along the orthogonal transverse axis whose strength is approximately linear in the arrival time of the particles. As a result, the longitudinal distribution of the beam is deflected transversely and can be imaged on a simple profile monitor located downstream [3]. This process is illustrated in Fig 2. The final transverse RMS beam size along the deflecting axis after a drift $L$ following the cavity is given by $\sigma_{x}=\sqrt{\sigma_{0}^{2}+\sigma_{d e f}^{2}}$ where $\sigma_{0}$ is the beam size with the deflector off and [4]

$$
\sigma_{d e f}=2 \sigma_{z} L \frac{\pi f V_{0}}{c U / e}
$$

where $\sigma_{z}$ is the RMS bunch length, $f$ is the RF frequency, $V_{0}$ is the deflecting voltage, and $U$ is the beam energy. The achievable temporal resolution can then be written as

$$
\Delta t=\Delta x \frac{U / e}{L \pi f V_{0}},
$$

where $\Delta x$ is the achievable spatial resolution of the profile monitor and the camera and optics used to view it. The transverse voltage is related to the input RF power $P$ by $V_{0}=\sqrt{n R_{T} P}$ where $n$ is the number of cells and $R_{T}$ is the transverse shunt impedance per cell. Based upon various spatial and hardware concerns, we have chosen a 9-cell 
standing-wave $\pi$-mode structure operating at a frequency of $9.59616 \mathrm{GHz}$. This gives an estimated shunt impedance of $680 \mathrm{k} \Omega$ per cell and a deflecting voltage of $V_{0}=550 \mathrm{kV}$ at an input power of $P=50 \mathrm{~kW}$. At this voltage, the temporal resolution given by Eq. (2) is on the order of $50 \mathrm{fs}$. The 9-cell structure was designed using the RF modeling codes MAFIA and HFSS 9.0. An image of the cavity geometry from these simulations and a plot of the (magnetic) field profile are shown in Fig. 3, followed by a list of parameter values in Table 1.

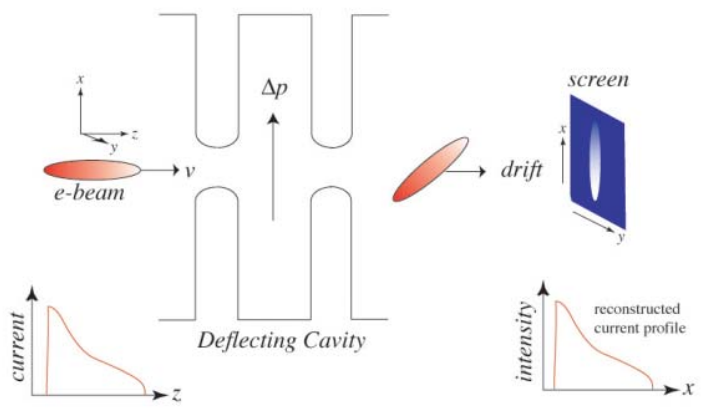

Figure 2: Illustration of the deflection of a beam by a dipole mode cavity phased at the zero-crossing of the RF.

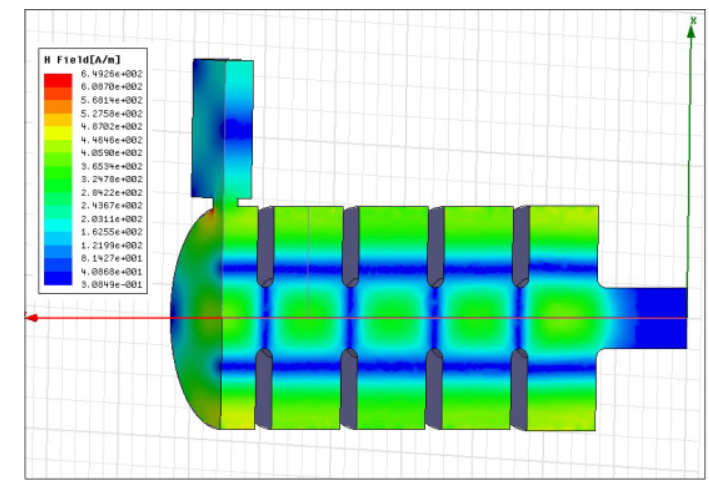

Figure 3: Plot of deflecting mode cavity geometry (quarter view) and axial transverse magnetic field profile from the center cell to the exit of the structure from HFSS simulations (quarter view).

Due to the presence of the irises which couple the cells, there is a nonzero transverse electric field in the vicinity of each iris. However, for a $\pi$-mode structure, the electric field produces a net deflection in the same direction as the magnetic field and thereby enhances the deflection of the beam.

\section{STEEL PROTOTYPE RESULTS}

The stainless steel cavity prototype design incorporates a knife-edge vacuum seal machined directly into the mating faces of the cells, which can accommodate a copper gasket or o-ring. This allows the cavity to be easily disassembled,
Table 1: Cavity Design Parameters

\begin{tabular}{|l|c|c|}
\hline Parameter & Value & Units \\
\hline Number of Cells & 9 & - \\
Pi-Mode Freq & 9.59616 & $\mathrm{GHz}$ \\
Transverse Shunt Impedance & 6.1 & $\mathrm{M} \Omega$ \\
Deflecting Voltage & 552 & $\mathrm{kV}$ \\
Quality Factor & 9080 & - \\
Cell Radius & 18.25 & $\mathrm{~mm}$ \\
Cell-to-Cell Distance & 15.62 & $\mathrm{~mm}$ \\
Iris diameter & 10 & $\mathrm{~mm}$ \\
Beam pipe diameter & 10 & $\mathrm{~mm}$ \\
VSWR & 1.0 & - \\
\hline
\end{tabular}

and will also permit a vacuum test of the prototype without the need for brazing or welding, which can warp and detune the cells. A drawing of the assembled prototype cavity is shown in Fig. 4 with a quarter section removed to reveal the interior of the structure. The inner RF surfaces of the structure were electrolytically coated with a 1 micron thick layer of copper.

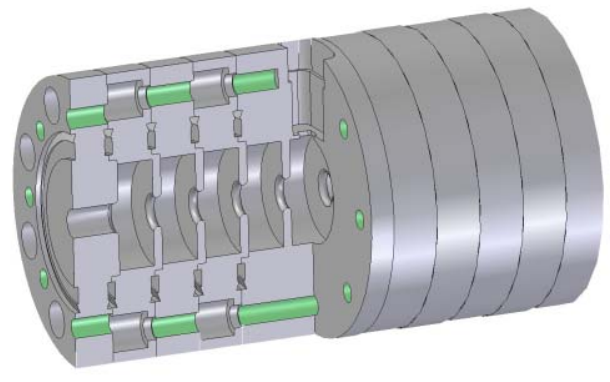

Figure 4: Cutaway drawing of the assembled 9-cell cavity design.

Note that the prototype dimensions are scaled to a slightly lower frequency than the final cavity design, due to the fact that the frequency had not yet been finalized at the time of its construction. This can be seen in the $S_{11}$ frequency scan in Fig. 5. Only five resonances appear, indicating that the intermediate modes corresponding to odd multiples of $\pi / 8$ are suppressed by the choice of a central coupler. The predicted HFSS frequency for the $\pi$-mode for this geometry (in air) is $9.4953 \mathrm{GHz}$. The $\pi$-mode frequency lies within $5 \mathrm{MHz}$ of the simulation prediction. Final frequency tuning will be accomplished by heating the cavity with a PID-feedback temperature control system. The estimated frequency correction factor is $d f / d T=-150 \mathrm{kHz} / \mathrm{C}$ for steel and $-142 \mathrm{kHz} / \mathrm{C}$ for copper.

It can be seen in Fig. 5 that there is good mode separation between the $\pi$-mode resonance and the nearest nonsuppressed mode. In addition, the VSWR is very close to unity. The depth of the $\pi$-mode resonance is not fully apparent in Fig. 5 due to the limited resolution of the plot, but it actually extends to about $-50 \mathrm{~dB}$. 


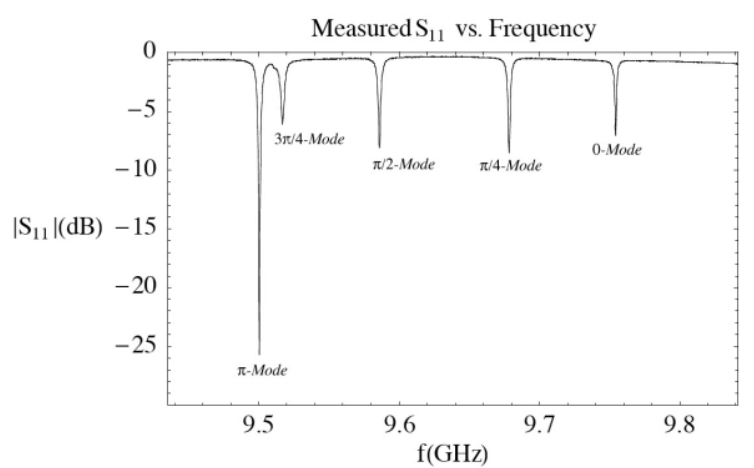

Figure 5: Plot of the RF reflectance at the input coupler of the prototype cavity showing the 5 non-suppressed resonances.

Table 2: Measured Prototype Parameters (in Air)

\begin{tabular}{|l|c|c|}
\hline Quantity & Meas & Units \\
\hline$f_{\pi}$ & 9.5001 & $\mathrm{GHz}$ \\
$f_{3 \pi / 4}$ & 9.5169 & $\mathrm{GHz}$ \\
$\Delta f$ & 16.9 & $\mathrm{MHz}$ \\
VSWR & 1.006 & - \\
$Q_{0}$ & 7839 & - \\
\hline
\end{tabular}

A bead pull was also performed on the prototype using an uncalibrated aluminum bead. The resulting shift in frequency as a function of the bead position along the cavity axis was measured using a network analyzer. By Slater's perturbation formula, the frequency shift observed is related to the field amplitudes $E_{0}$ and $H_{0}$ along the axis by the formula [8]

$$
\frac{\Delta f}{f_{0}}=\frac{\pi a^{2}}{W_{0}}\left(\left|E_{0}\right|^{2}-\frac{1}{2}\left|H_{0}\right|^{2}\right)
$$

where $a$ is the bead diameter, and $W_{0}$ is the stored energy in the cavity.

Consequently, the positive peaks in Fig. 6 correspond with the irises where $E_{0}$ is maximum, and the negative peaks correspond with the centers of the cells where $H_{0}$ is

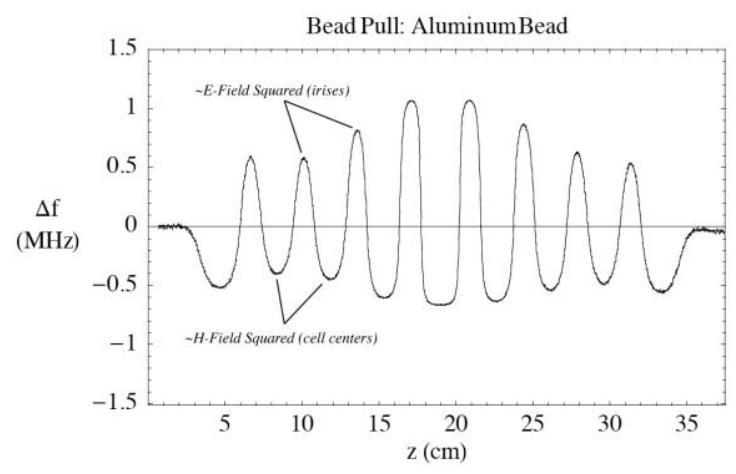

Figure 6: Bead pull field measurement of the prototype cavity using a metallic bead. maximum. The field balance is imperfect due to difficulties involved in attempting to tune the cells of a steel cavity for a copper-based design prior to application of the 1-micron copper coating. Also note that the flattening of the peaks near the center cell is a systematic error due to the fact that the frequency deviation in this region approaches the width of the resonance. This may be corrected in the future by using a smaller bead.

\section{CONCLUSIONS}

The final cavity will be constructed of solid copper using the design parameters of Table 1 . This design is expected to provide temporal resolution of the electron beam at the 50 fs level, which should be sufficient to resolve the picosecond to sub-picosecond current profiles generated by the UCLA Neptune accelerator and dogleg compressor beamline. The cell-to-cell mating will incorporate the vacuum seal design of the steel prototype, in order to simplify the process of assembling and tuning the cavity, but with viton o-rings between the mated cells. An alternative hybrid design using copper cells with stainless steel knife-edges is currently being explored for applications with more stringent vacuum requirements.

\section{REFERENCES}

[1] P. R. Phillips. The Separation of High-Energy Particle Beams by Microwave Techniques. $\mathrm{PhD}$ thesis, Stanford University, Stanford, CA 94305, November 1960.

[2] O. A. Altenmueller, R. R. Larsen, and G. A. Loew. Investigations of Travelling-Wave Separators for the Stanford TwoMile Linear Accelerator. Technical Report PUB-R-017, Stanford Linear Accelerator Center, Stanford, CA, 1963.

[3] G. A. Loew and O. H. Altenmueller. Design and Application of RF Separator Structures at SLAC. Technical Report PUB135, Stanford Linear Accelerator Center, Stanford, CA, 1965.

[4] P. Emma, J. Frisch, and P. Krejcik. A Transverse RF Deflecting Structure for Bunch Length and Phase Space Diagnostics. Technical Report LCLS-TN-00-12, Stanford Linear Accelerator Center, Stanford, CA, 2000.

[5] J. Haimson. Longitudinal phase space measurements of short electron bunches using a $17 \mathrm{ghz}$ circularly polarized beam deflector. In Advanced Accelerator Concepts: Eleventh Workshop, pages 95-108, 2004.

[6] Derun Li and J. N. Corlett. Deflecting rf cavity design for a recirculating linac based facility for ultrafast $\mathrm{x}$-ray science (lux). In Proceedings of the 2003 Particle Accelerator Conference, pages 1249-1251, 2003.

[7] R. J. England, J. B. Rosenzweig, G. Andonian, P. Musumeci, G. Travish, and R. Yoder. Sextupole Correction of the Longitudinal Transport of Relativistic Beams in Dispersionless Translating Sections. Phys. Rev. ST-AB, 8:12801, January 2005.

[8] J. C. Slater. Microwave Electronics. Van Nostrand, Princeton, NJ, 1950 